\title{
Trends in Automotive Exhaust Gas Sensing
}

\author{
Classen, Thomas; Sahner, Kathy \\ Corporate Research, Robert Bosch $\mathrm{GmbH}$, Stuttgart \\ Postfach 1060 50, 70049 Stuttgart
}

\section{Introduction}

In 1976 Bosch introduced the switching type lambda sensor into the automotive market. Since then the field of exhaust gas sensors has been growing continuously. In 1998 an improved sensor type, the broadband oxygen sensor, was introduced into series production allowing measuring the oxygen concentration in the exhaust over a wide range. The broader measuring range and the high signal dynamics enable continuous system control in contrast to the two position control of the switching type sensor. While lambda sensors are standard in all gasoline systems, the introduction of gas sensors into diesel systems is continuously progressing. The combination of energy efficient diesel engines with exhaust gas aftertreatment allows $\mathrm{CO}_{2}$ and emission efficient mobility. Sensors play an import role in the control and diagnosis of the involved aftertreatment components. The wideband lambda sensor was introduced to Diesel systems in 2002 enabling improved engine control and emission reduction. Additionally, modern Diesel systems employ a variety of other exhaust sensors, namely temperature sensors and pressure sensors. The control of Diesel particulate filters (DPF) requires measuring the differential pressure across the filter. Future diesel systems may additionally include particulate sensors for direct DPF diagnosis. To reduce the emission of nitrous oxides $\left(\mathrm{NO}_{x}\right)$ additional catalysts require Lambda $\left(\mathrm{NO}_{x}\right.$ Storage Catalyst / NSC) or $\mathrm{NO}_{x}$-Sensors (Selective Catalytic Reduction Systems / SCR). The combination of these exhaust gas sensors and components allow minimizing the exhaust emissions at low fuel consumption levels.

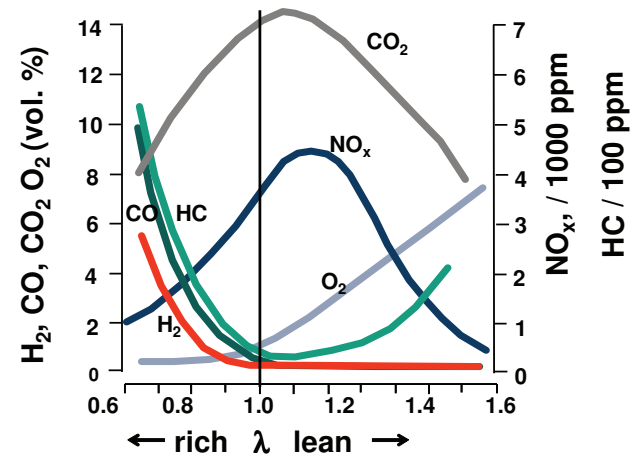

Figure 0: Emission control by lambda measurement.

\section{Lambda sensors}

In gasoline cars, switching type lambda sensors offer high precision determination of lambda values close to stoichiometric burning conditions. For the fast and reliable lambda determination across the full lambda range advanced amperometric wideband oxygen sensors are necessary. The combination of both sensor types enables a close circuit operation with both fast response and high accuracy.

For the sensor elements of lambda sonds the zircon dioxide technology has been proven to be perfectly suited. Yttria stabilized Zirconia both exhibits high rigidity as well as long term stability in corrosive environemt. Oxygen ion conductivity takes place above $300^{\circ} \mathrm{C}$ between platinum electrodes. A Nernst voltage is observed between two electrodes of an electrochemical Nernst cell in different oxygen atmosphere that depends critically on the relative oxygen partial pressures. Having one electrode at $\lambda=$ 1 a voltage drop by several orders of magnitude is observed against the reference electrode. This enables a reliable engine control to this point of stoichiometric combustion characterized by low total emission. 
For a wideband oxygen sensor a second electrochemical cell is used. By actively applying a voltage to this cell, oxygen is "pumped" through the zircon dioxide. A prorous difusion barrier in front of one electrode of this pumping cell limits the transport of oxygen gas molecules to the cell. The measured current across this cell is then proportional to oxygen transport across the diffusion barrier, which in turn is proportional to the overall oxygen content in the gas. A closed feedback loop for the pumping voltage secures the accuracy of the sensor signal and enables measurement in rich gas. In this way, a wideband oxygen sensor can measure the oxygen concentration in a continuous manner across a wide concentration regime.

According to the further tightening emission legislation the development of the Lambda sensors is constantly progressing. The first sensor generation depended on clean reference air for accurate sensor signals. This necessity is avoided by an internal, so called pumped reference. Hence, the sensors creates his own pure oxygen reference.

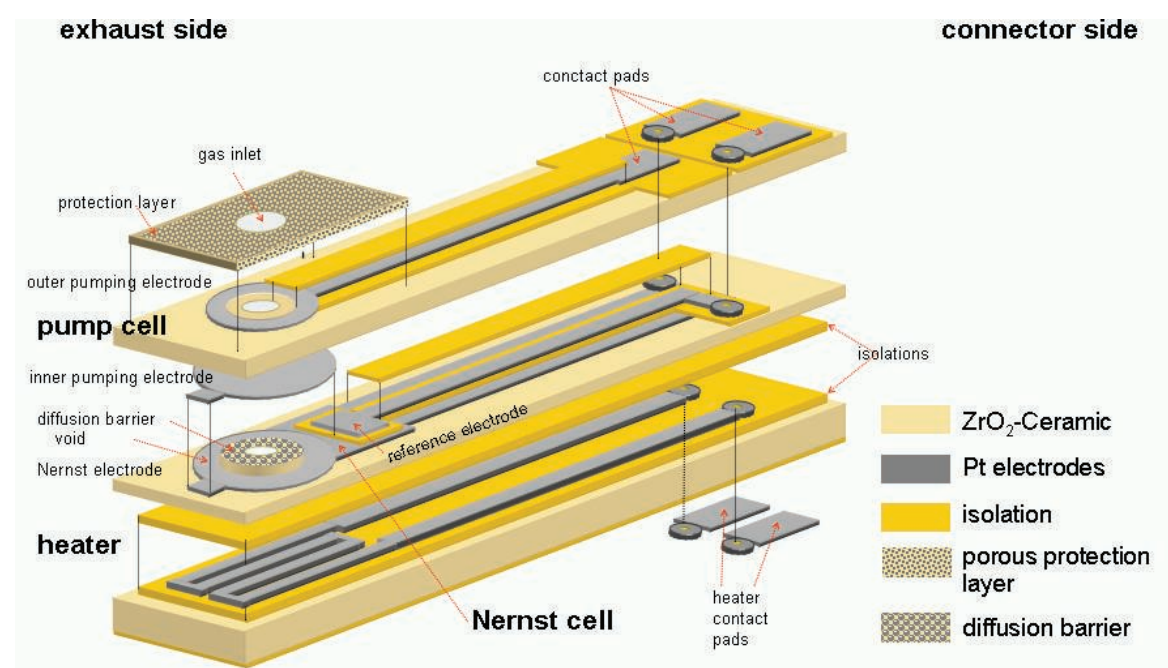

Figure 1: Wideband Oxygen Sensor LSU4.9

A fast sensor readiness after engine is strongly beneficial to reduce the total engine emission. As in the early phase after the engine start the exhaust gas and its pipe system are still cold, water droplets exists in the exhaust gas. The ceramic sensor element has to be protected against the thermal shock of a water droplet on the hot sensor element. A thermal shock protection layer can significantly reduce this impact and thus allows the use the lamba sensor as fast as 10 seconds after engine start allowing an early start of exhaust gas conversion at the catalyst.

The advantages of using wide band oxygen sensors in Gasoline cars are the following:

Port Fuel Injection (PFI):

- $\lambda=1$ : Improvement of the conversion of the Three-Way-Catalyst (TWC), reduced size of catalyst possible, single cylinder control

- $\quad \lambda>1$ : lean run with reduced emissions

- $\lambda<1$ : rich run, reduction of fuel consumption

- diagnosis

$\circ$ of TWC: measuring the $\mathrm{O}_{2}$-storage capacity that is directly correlated with the cat activity $\circ$ of the secondary air injection for fast catalyst heating

Direct Injection (as above plus):

- $\quad \lambda>1$ (homogen): reduced $\mathrm{NO}_{x}$ raw emission and consumption

- $\lambda<1$ : regeneration of $\mathrm{NO}_{x}$-storage-catalyst

\section{Other Exhaust Gas Sensors}

Since 2002 the wideband lambda sensor is used in Diesel passanger cars to reduce the emissions. The LSU is used both for the adaption of the mass meanvalue adaption and for the fuel mass observer. Both applications are used to run the engine in optimized areas of the parameter field avoiding for example the smoke burst during accelleration. Current Diesel systems additionally use several other exhaust gas sensors, namely temperature sensors, pressure sensors and $\mathrm{NO}_{x}$-sensors. 


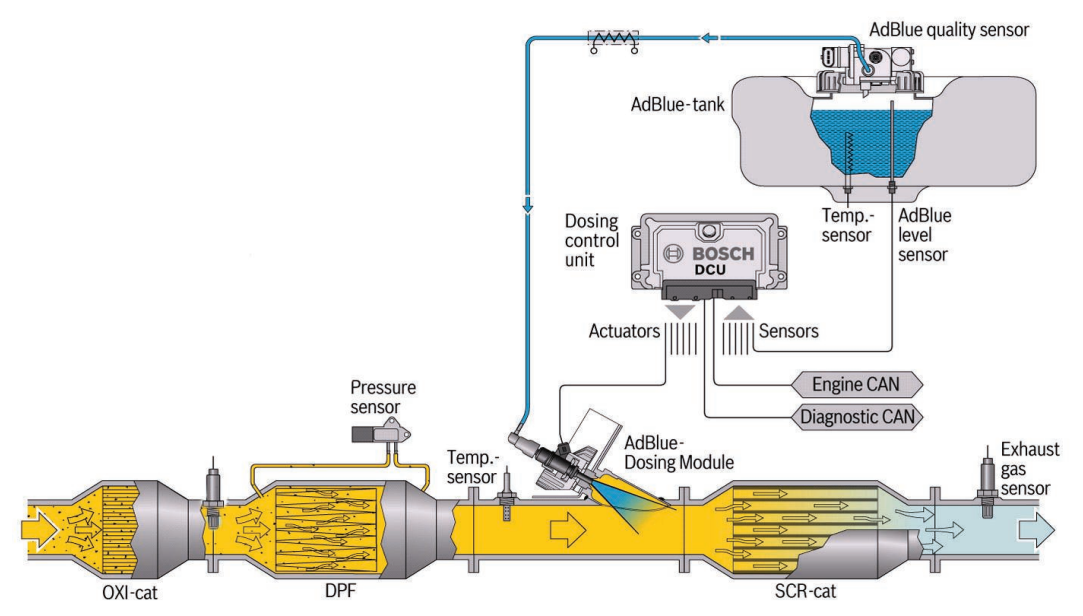

The constant trend to reduce emissions has brought the necessity to include further components for exhaust gas aftertreatment into a Diesel exhaust pipe.

The Diesel particulate filter (DPF) withholds particulate emissions by accumulation of particulate matter on its porous ceramic wall structure. For monitoring its degree of loading and for diagnosis a differential pressure sensor is necessary comparing the relative pressure before and after the DPF. For running a regeneration but also for engine control various temperature sensors are employed.

The Diesel Oxidation Catalyst (DOC) allows to react unburned fuel components, which is both necessary for limiting hydrocarbon $(\mathrm{HC})$ emissions and for DPF regeneration. It also partially converts $\mathrm{NO}$ into $\mathrm{NO}_{2}$, which is necessary for efficient operation of the so called Selective Catalyst Reaction (SCR)-Cat.

In the SCR, $\mathrm{NO}, \mathrm{NO}_{2}$ and $\mathrm{NH}_{3}$ react to form water and $\mathrm{CO}_{2}$. The $\mathrm{NH}_{3}$ for this process is supplied by urea water mixtures ("Ad-Blue") dosed into the exhaust pipe system. For dosing control and for monitoring the conversion $\mathrm{NO}_{x}$-sensors are employed.

An alternative to the SCR catalyst is the NO-Storage Catalyst (NSC) which can be operated and monitored by Lambda sensors.

A NO $\mathrm{N}_{\mathrm{x}}$ sensor can be seen as the next step in complexity of a Zirconia based sensor beyond a Lambda sensor. It consists of two chambers with different electrochemical cells. In its front cell, it operates in a similar principle as the wideband lambda sensor, pumping oxygen out of a chamber behind a diffusion barrier. Sequencial to this step, the remaining gas is fed into a second chamber by means of a second diffusion barrier. Here the next gas component in the electrochemical voltage series is reduced - the NO. The oxygen which results from this process is electrochemically pumped away giving rise to a current that is proportional to the $\mathrm{NO}_{x}$ concentration.

\section{Future Trends and Outlook}

There is a constant trend to improve the fuel and $\mathrm{CO}_{2}$ economy of automobiles while at the same time further reducing the emissions of toxic species such as $\mathrm{CO}$ or $\mathrm{NO}_{x}$. The introduction of electrical vehicles which locally emit no toxic gases at all will enforce this trend. Among the vehicles with internal combustion engines those that have best fuel economy with the lowest toxic emissions will be able to best stand the direct comparison.

Additional needs arise from the legal requirements to monitor all exhaust components to secure their function over lifetime. As the total number of sensors has to be limited for economical reasons, the reliability and accuracy of the extending sensors will be further increased to improve the overall performance of the exhaust gas aftertreatment. The next steps in emission legislation will be accompanied by a further penetration of new exhaust gas sensors into automotive systems. 